\title{
THE NATURE OF THE DENSE CORE POPULATION IN THE PIPE NEBULA: THERMAL CORES UNDER PRESSURE
}

\author{
Charles J. Lada, ${ }^{1}$ A. A. Muench, ${ }^{1}$ J. Rathborne, ${ }^{1}$ João F. Alves,${ }^{2}$ and M. Lombardi ${ }^{3,4}$ \\ Received 2007 May 24; accepted 2007 September 4
}

\begin{abstract}
In this paper we present the results of a systematic investigation of an entire population of predominately starless dust cores within a single molecular cloud, the Pipe Nebula. Analysis of extinction data shows the cores to be dense objects characterized by a narrow range of density with a median value of $n\left(\mathrm{H}_{2}\right)=7 \times 10^{3}$. The nonthermal velocity dispersions measured in molecular emission lines are found to be subsonic for the large majority of the cores and show no correlation with core mass (or size). Thermal pressure is found to be the dominate source of internal gas pressure and support for most of the core population. The total internal pressures of the cores are found to be roughly independent of core mass over the entire $\left(0.2-20 M_{\odot}\right)$ range of the core mass function (CMF) indicating that the cores are in pressure equilibrium with an external source of pressure. This external pressure is most likely provided by the weight of the surrounding molecular cloud. Most of the cores appear to be pressure confined, gravitationally unbound entities whose fundamental physical properties are determined by only a few factors, which include self-gravity, gas temperature, and the simple requirement of pressure equilibrium with the surrounding environment. The entire core population is found to be characterized by a single critical Bonnor-Ebert mass of approximately $2 M_{\odot}$. This mass coincides with the characteristic mass of the Pipe CMF suggesting that the CMF (and ultimately the stellar IMF) has its origin in the physical process of thermal fragmentation in a pressurized medium.
\end{abstract}

Subject heading: stars: formation

Online material: color figures

\section{INTRODUCTION}

The stellar initial mass function (IMF) is one of the most fundamental distributions in astrophysics. Its origin is one of the most critical but least understood aspects of the star-forming process and is perhaps the most fundamental unsolved problem of star formation (e.g., Corbelli et al. 2005; Bonnell et al. 2007). Stars form in the dense cores of molecular clouds, but little is understood about the detailed physical properties of these cores prior to star formation and even less is understood about their origin. However, both these issues are critically linked to the origin of the IMF.

Lack of even a rudimentary understanding of these issues stems primarily from a general lack of empirical knowledge of earliest stages of dense core evolution. This state of affairs is a result of an absence of molecular clouds identified in a sufficiently early evolutionary state that little or no star-forming activity is present within them. This is often taken as evidence that the onset of star formation in molecular clouds is extremely rapid and that clouds in the earliest evolutionary states must be exceedingly rare. Clearly the identification and detailed study of such a cloud and its dense core population would be of great interest.

We have identified the visually prominent dark cloud known as the Pipe Nebula as one of the best candidates for an extremely young molecular cloud complex. At a distance of $130 \mathrm{pc}$ (Lombardi et al. 2006) this cloud is one of the nearest objects of its mass $\left(\sim 10^{4} M_{\odot}\right)$ and size $(\sim 3 \times 14 \mathrm{pc})$ to the Sun. The cloud exhibits very little evidence of star formation activity, and this is probably

\footnotetext{
${ }^{1}$ Harvard-Smithsonian Center for Astrophysics, 60 Garden Street, Cambridge, MA 02138; clada@cfa.harvard.edu.

2 Calar Alto Observatory, Centro Astronómico Hispano Alemán, c/Jesús Durbán Remón 2-2, 04004 Almeria, Spain; jalves@caha.es.

3 European Southern Observatory, Karl-Schwarzschild-Strasse 2, 85748 Garching, Germany.

${ }^{4}$ University of Milan, Department of Physics, via Celoria 16, 20133 Milan,
}

reflected in the fact that prior to 2006 only one paper in the literature was devoted to its study. That paper, a CO survey by Onishi et al. (1999), showed that despite the paucity of star formation activity the cloud was similar enough in its ${ }^{12} \mathrm{CO}$ and ${ }^{13} \mathrm{CO}$ emission to the Taurus clouds that its potential to form stars was high.

These considerations motivated us to undertake a detailed and systematic multiwavelength investigation of this cloud in order to quantify its overall properties. The primary goal of this program was to identify the complete population of its dense cores and then determine their detailed physical nature. The study of the core population in a single cloud ensures that all the cores are at the same distance, removing one of the largest sources of systematic uncertainty in relative comparisons of core physical properties. The proximity of the Pipe cloud is critical to enable even the smallest and lowest mass cores to be detected and resolved. In the first step of this investigation we obtained a wide-field, nearinfrared extinction map of the entire $6^{\circ} \times 8^{\circ}$ extent of the Pipe region and quantified the basic structure and properties of the cloud (Lombardi et al. 2006). Using that data, we then identified its core population and determined their masses (Alves et al. 2007). The dust extinction measurements enabled robust determination of core masses over a large dynamic range $\left(0.2-20 M_{\odot}\right)$ with more statistically significant sampling to lower masses than previously achieved using different techniques in other clouds. The Pipe cores appear to be a pristine population of starless objects whose mass spectrum displays an overall shape (and width) nearly identical to that of the stellar IMF, but offset in mass by a factor of 3 . These observations suggested that the core mass function (CMF) of the Pipe Nebula is the direct precursor to the stellar IMF, once it is modified by a constant star formation efficiency (SFE) of $\sim 30 \%$.

Determination of the detailed physical properties of such a population of (mostly starless) dense cores cannot only provide important information about the initial conditions of star formation 
within the individual cores, but also potentially new insights concerning the nature and the origin of the CMF and perhaps even the IMF itself. Our measurements of dust extinction can provide robust determinations of fundamental core properties such as the sizes, masses, and densities. To obtain a complete description of basic core properties, however, requires direct observations of the gas within the cores. Observations of molecular lines can provide critical information about both the overall kinematics and the internal dynamical states of dense cores. This information in turn enables determinations of the boundedness and stabilities of the cores, as well as the internal pressures characterizing them. Therefore, in an effort to obtain a more complete physical description of the core population in the Pipe cloud, we augmented our extinction survey with two molecular emission line surveys of the core population. The first survey was a $\mathrm{C}^{18} \mathrm{O}$ survey of a large fraction $\left(\frac{2}{3}\right)$ of the cores designed to provide information about the kinematics and dynamical nature of as complete as sample of the cores as possible (Muench et al. 2008). This was followed by a directed $\mathrm{NH}_{3}$ study of a smaller but significant sample of the core population to measure the conditions in the densest material (Rathborne et al. 2008). In this paper we combine the results molecular-line surveys with the extinction observations to perform a detailed examination of the basic physical properties of this important core population.

\section{RESULTS AND ANALYSIS}

\subsection{Dense Cores}

Alves et al. (2007, hereafter ALL07) used a wavelet decomposition technique to identify 159 cores in the Pipe cloud from the wide-field extinction map of Lombardi et al. (2006, hereafter LAL06). The wavelet decomposition effectively acts as a low pass filter to remove a relatively extended smoothly varying background from the extinction image. This results in the construction of a "cores only" image of the small-scale $(l \leq 0.3 \mathrm{pc})$ spatial structure in the cloud. Visual inspection of this image showed a population of well-defined and well-separated extinction peaks or cores. An automated two-dimensional algorithm (Clumpfind2d; Williams et al. 1994) was used to systematically identify and extract cores in an objective manner. The detection threshold was set at $A_{V}=1.2 \mathrm{mag}$, which is three times the rms variation in extinction over extended regions of the image nearby but off the cloud and 6 times the measured rms uncertainty in individual pixels of the original extinction image (LAL06). The input parameters for the algorithm were adjusted to minimize and eliminate spurious core identifications. This requirement was met by demanding visual verification of each core extracted. The primary advantage of using this technique is to produce a systematic and objective measurement of the area, $A$, of each core which can then be converted to a radius via $R=(A / \pi)^{1 / 2}$. This radius corresponds to the outer edge of the core. The mass of a core is then calculated by integrating the (background-subtracted) dust column density over the area of the core and multiplying by an assumed gas-to-dust ratio (in this case a ratio of 100).

Because the uncertainties in the extinction measurements are extremely small (on the order of a few percent), the primary source of systematic uncertainty in the derived masses (apart from the uncertainty in the distance to the cloud) is the uncertainty in the derived area of an extracted core. To estimate this uncertainty we performed a number of core extractions varying the input parameters, including the threshold extinction while relaxing somewhat the visual verification constraint. We then compared the results for individual cores identified in common. From these experiments we estimate the typical uncertainty in core area (and

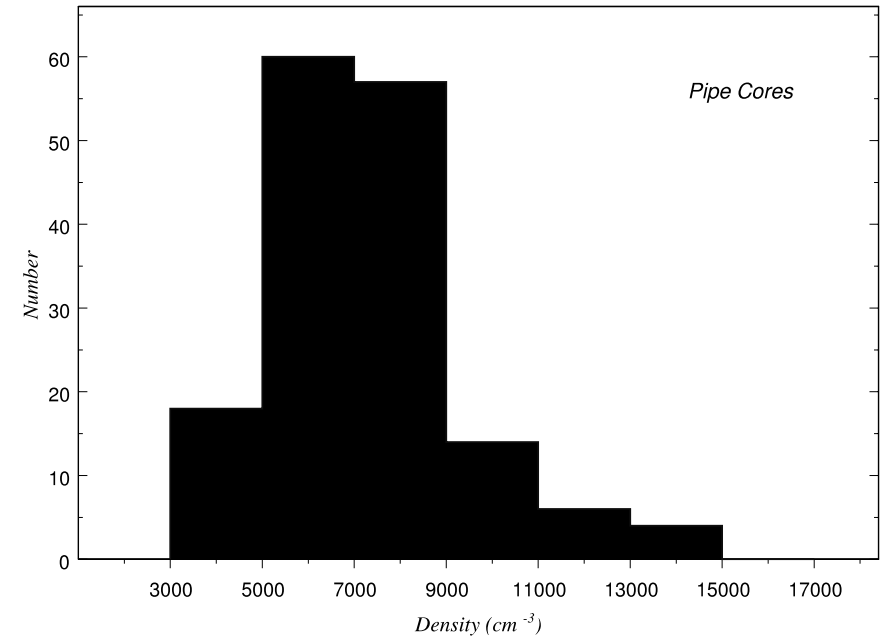

FIG. 1.-Frequency distribution of mean core particle densities $\left(\mathrm{cm}^{-3}\right)$. The extinction cores identified in the Pipe cloud are dense cores with a relatively narrow spread in mean density. [See the electronic edition of the Journal for a color version of this figure.]

mass) to be between $10 \%$ and $30 \%$. Thus the extracted core radii and derived masses appear to be quite robust.

With good masses and radii in hand we can then calculate the mean densities of each core, assuming spherical symmetry, from $\rho=3 M /\left(4 \pi R^{3}\right)$. The corresponding volume number density is $n\left(\mathrm{H}_{2}\right)=\rho / \mu m_{\mathrm{H}}$, where $\mu$ is the molecular weight (2.34, for a molecular hydrogen gas) and $m_{\mathrm{H}}$ is the mass of a proton. In Table 1, in the Appendix, we list the radii, masses, and densities determined for the cores in the Pipe Nebula.

Figure 1 displays the frequency distribution of core number densities in the Pipe cloud. The core density distribution is characterized by a well-defined and relatively narrow peak. The mean core density is found to be $7.3 \times 10^{3} \mathrm{~cm}^{-3}$. The dispersion in density is $2 \times 10^{3} \mathrm{~cm}^{-3}$. The distribution is not perfectly symmetric about this mean and displays a significant tail to higher densities. The median density of the cores is found to be $7.1 \times 10^{3} \mathrm{~cm}^{-3}$. As discussed by Rathborne et al. (2007), this is consistent with the $\mathrm{NH}_{3}$ emission exhibited by the core population in the Pipe cloud. Although the typical density of the Pipe cores is somewhat lower than that of a few $\times 10^{4} \mathrm{~cm}^{-3}$ usually attributed to cores in other clouds (e.g., Jijina et al. 1999), the core population identified by ALL07 and investigated here is a population of dense cores.

\subsection{Thermally Dominated Cores}

Molecular emission-lines provide important information about the kinematics and internal dynamics of molecular clouds. A little over a quarter century ago Larson (1981) used observations of CO to establish that molecular clouds appear to satisfy three general relations, now known as Larson's laws. The first of these laws expresses a relation between the size of a cloud and its velocity dispersion, namely, $\sigma \sim R^{0.5}$. On the scales of dense cores $\sigma$ is determined directly from the line width. The second law was that of approximate virial equilibrium, $\left(\sigma^{2} R\right) /(G M) \sim 1$. The third law, which follows from the first two, relates the sizes and masses of the clouds and indicates that clouds are characterized by constant column density, that is, $M \sim R^{2}$. These laws are widely interpreted to indicate that the physical state of molecular clouds is best described by a common hierarchy of (supersonic) turbulent motions under the influence of gravity (Larson 1981).

Figure 2 plots the one-dimensional nonthermal velocity dispersions, $\sigma_{\mathrm{NT}}$, in the individual Pipe Nebula cores versus core 


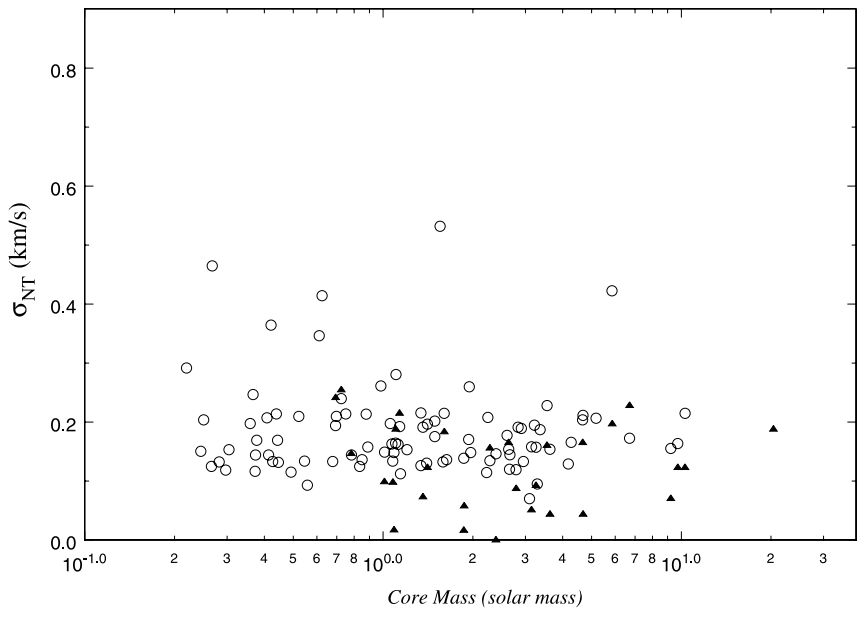

FIG. 2.- Relation between nonthermal velocity dispersion and mass for the dense core population of the Pipe Nebula. These dispersions are predominately subsonic and not correlated with core mass. Open circles correspond to $\mathrm{C}^{18} \mathrm{O}$ measurements, and the filled triangles to $\mathrm{NH}_{3}$ measurements. [See the electronic edition of the Journal for a color version of this figure.]

mass for both $\mathrm{C}^{18} \mathrm{O}$ and $\mathrm{NH}_{3}$ observations. These velocity dispersions were calculated from the molecular line widths assuming a gas kinetic temperature of $10 \mathrm{~K}^{5}$ There is no correlation between nonthermal velocity dispersion and core mass. Moreover, the vast majority of these velocity dispersions have magnitudes less than the speed of sound, $0.2 \mathrm{~km} \mathrm{~s}^{-1}$, in a $10 \mathrm{~K}$ molecular gas. The nonthermal motions in these cores are thus subsonic across the entire spectrum of core masses in the cloud, a spectrum which covers nearly 2 orders of magnitude in mass. This suggests that the gas motions in the cores are acoustic and thermally dominated. A very important consequence of this finding is that the cores in the Pipe Nebula must evolve on acoustic and thus relatively slow timescales. The typical core sound crossing time is about $10^{6} \mathrm{yr}$.

The results of Figure 2 coupled with the absence of a sizeline width relation in both the $\mathrm{C}^{18} \mathrm{O}$ and $\mathrm{NH}_{3}$ lines for the Pipe core population (Muench et al. 2008; Rathborne et al. 2008) indicates that this population violates Larson's laws for turbulent molecular clouds. If the nonthermal motions in the Pipe cores are due to turbulence, this turbulence is of a different nature than that which is typically described by Larson's laws. It is subsonic, rather than supersonic, and is characterized by a scale that is smaller than the core size and thus it is more likely microturbulent rather than macroturbulent in nature.

To further investigate the possibility of thermally dominated motions in the Pipe cores, we consider the ratio of thermal to nonthermal pressure i.e., $R_{p}=a^{2} / \sigma_{\mathrm{NT}}^{2}$, for each core; here $a$ is the one-dimensional isothermal sound speed in a $10 \mathrm{~K}$ gas. In Figure 3 we plot $R_{p}$ versus mass. For more than two-thirds of the cores measured in $\mathrm{CO}$ and $80 \%$ of the cores measured in $\mathrm{NH}_{3}$ $R_{p}>1$ and the thermal pressure clearly exceeds the nonthermal pressure. Indeed, in the most extreme cases, the thermal pressure is 1-2 orders of magnitude higher than the nonthermal pressure. For three of these cores the observed $\mathrm{NH}_{3}$ line widths were indistinguishable from purely thermally broadened line profiles in

\footnotetext{
5 Rathborne et al. (2008) were able to estimate kinetic temperatures for 12 cores and found that higher mass cores, with the strongest $\mathrm{NH}_{3}$ detections were characterized by temperatures $\sim 10( \pm 1) \mathrm{K}$, while lower mass cores with weaker detections were characterized by temperatures of $\sim 13( \pm 3) \mathrm{K}$. For the purposes of this paper we conservatively adopt a single kinetic temperature of $10 \mathrm{~K}$ for all the cores.
}

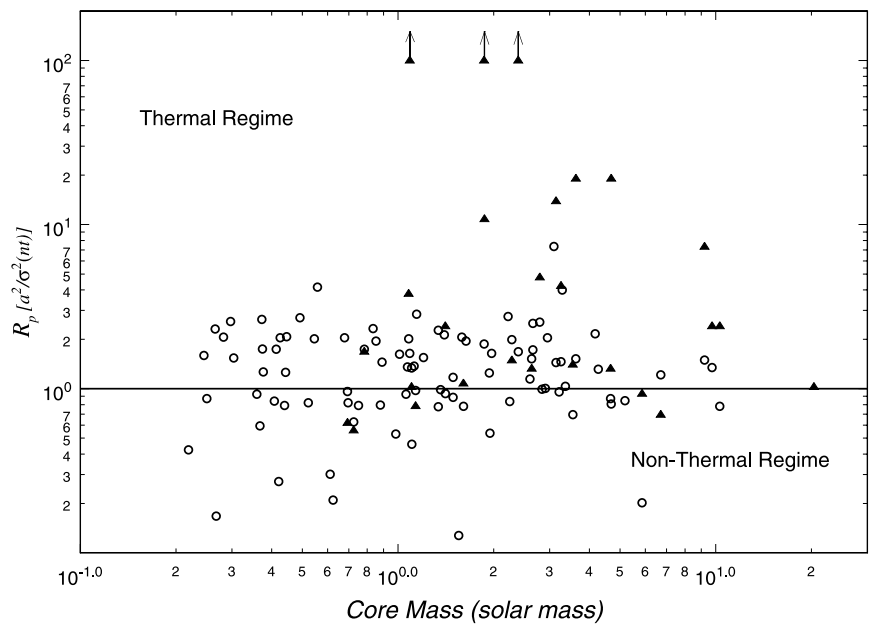

FIG. 3.- Ratios of thermal to nonthermal gas pressure in the Pipe cores. Thermal pressure dominates the internal pressure for the great majority of the cores. Thermal pressure is a significant pressure source $\left(R_{p}>0.5\right)$ for nearly all $(\sim 90 \%)$ the core population. The three lower limits correspond to three cores whose $\mathrm{NH}_{3}$ line widths were indistinguishable from purely thermal profiles in a $10 \mathrm{~K}$ gas. The symbol key identical to that of Fig. 2. [See the electronic edition of the Journal for a color version of this figure.]

a $10 \mathrm{~K}$ gas and lower limits to $R_{p}$ are plotted. ${ }^{6}$ For $90 \%$ of the CO measurements and all the $\mathrm{NH}_{3}$ measurements $R_{P}>0.5$. Therefore, thermal pressure is a significant if not the dominant source of internal gas pressure for essentially the entire core population.

We note here that our calculated values of $R_{p}$ are likely underestimates to the true values for many sources. This is due to two reasons. First, our $\mathrm{NH}_{3}$ observations show that the cores in the Pipe can have temperatures as high as $12-15 \mathrm{~K}$ (Rathborne et al. 2007) and thus our assumption of $10 \mathrm{~K}$ for the typical gas temperature may underestimate the actual gas temperatures for some fraction of the objects. Second, for most cores the nonthermal pressures were calculated from the $\mathrm{C}^{18} \mathrm{O}$ observations and this could result in an overestimate of the nonthermal pressure since the $\mathrm{C}^{18} \mathrm{O}$ observations sample both core and more extended intercore or background gas. This is particularly true for the lower mass cores, where as much as half or more of the total line-of-sight column density sampled by the $\mathrm{C}^{18} \mathrm{O}$ observations arises outside the core. Indeed, for 25 cores (excluding the three sources with lower limits) where both $\mathrm{NH}_{3}$ and $\mathrm{C}^{18} \mathrm{O}$ are observed, the nonthermal pressure derived from the $\mathrm{CO}$ data is on average a factor of 4 larger than that measured by the ammonia line.

The results described here are consistent with and confirm those derived by Barranco \& Goodman (1998) and Goodman et al. (1998), who studied four dense cores in four different clouds and, similar to the findings presented here, found the cores to be characterized by subsonic nonthermal motions. They argued that dense cores represented regions of "coherence" in otherwise turbulent molecular clouds. Apparently the physical conditions that characterize the population of cores in the Pipe cloud may be typical of dense cores in general, at least in regions of low-mass star formation.

\subsection{Pressure-Confined Cores}

\subsubsection{Gravitational Binding}

In this section we investigate the integrity of the cores as persistent, coherent entities. First, we consider the gravitational

\footnotetext{
${ }^{6}$ These three cores could also be characterized by $T_{k}<10 \mathrm{~K}$, in which case
} $R_{p}$ could be somewhat lower than plotted. 


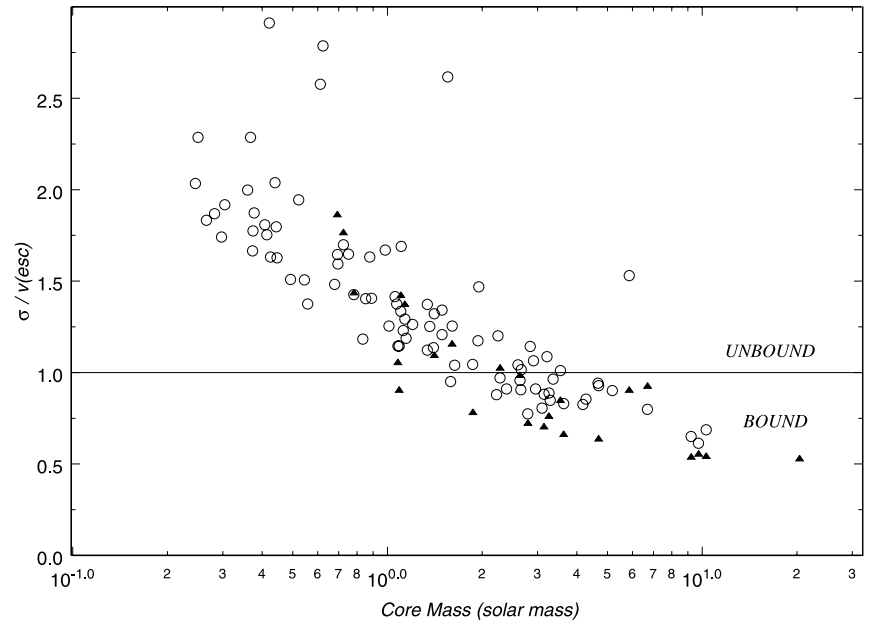

FIG. 4.- Ratio of total (three-dimensional) velocity dispersion to escape velocity plotted against core mass. Most cores in the Pipe cloud appear to be gravitationally unbound. The symbol key is identical to that of Fig. 2. [See the electronic edition of the Journal for a color version of this figure.]

binding of the cores. For each core we calculate the threedimensional velocity dispersion including both thermal and nonthermal contributions, i.e.,

$$
\sigma_{3 \mathrm{D}}=\sqrt{3 a^{2}+3 \sigma_{\mathrm{NT}}^{2}}
$$

and we compare this to the escape velocity of a spherical core with the same mass $(M)$ and size $(R)$, i.e,

$$
V_{\mathrm{esc}}=\sqrt{2 G M / R} .
$$

In Figure 4 we plot the ratio of $\sigma_{3 \mathrm{D}}$ to $V_{\mathrm{esc}}$ against the log of the core mass. This ratio of gas velocity dispersion to escape speed is well correlated with core mass. The majority of the cores appear to be unbound gravitationally. Thus these cores appear to violate the second of Larson's laws, that of gravitationally bound objects. The threshold between bound and unbound cores occurs at about $2-3 M_{\odot}$. It is interesting that this mass is similar to the mass at which the CMF breaks from a single power-law form before reaching its peak (ALL07). If they form stars, the bound cores will likely produce stars that populate the Salpeter (1955), power-law portion of the stellar IMF.

\subsubsection{Internal Pressures and Pressure Confinement}

What is the nature of the unbound cores? To investigate this further we calculated the average total (thermal+nonthermal) internal gas pressures of each of the Pipe cores, i.e.,

$$
P(\text { total })=P(T)+P(\mathrm{NT})=\rho\left(a^{2}+\sigma_{\mathrm{NT}}^{2}\right),
$$

where $\sigma_{\mathrm{NT}}$ is the one-dimensional, nonthermal velocity dispersion and $\rho$ is the mean density of a core calculated from the mass and size derived for it from the extinction data. In Figure 5 we plot the total pressure, $P$ (total $) / k$, versus core mass. The plot displays significant scatter (about a factor of 3 in pressure independent of core mass) and shows that the internal core pressure is not a particularly strong function of mass. Both the core pressure and spread in this pressure are surprisingly similar over the entire range of core mass. The facts that the cores are spread out over the entire $14 \mathrm{pc}$ length of the cloud and yet have very similar internal pressures, independent of whether they are $0.2-0.3$ solar mass

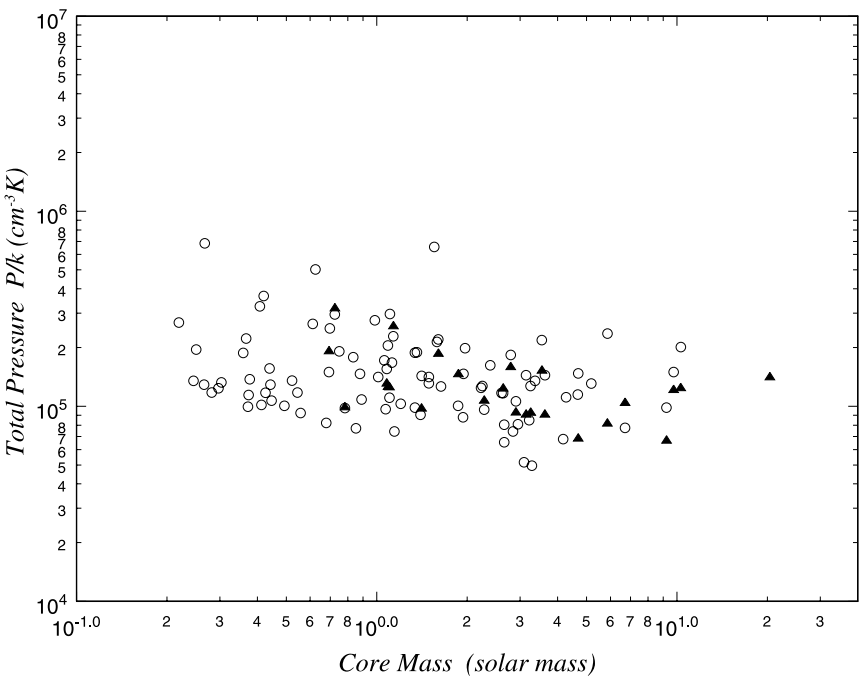

FIG. 5.- Total internal gas pressure plotted as a function of core mass. The close similarity of core pressures across the entire span of core mass suggests that the individual cores are in a state of pressure equilibrium with an external pressure source. Otherwise, symbols are the same as in Fig. 2. [See the electronic edition of the Journal for a color version of this figure.]

cores or 10-20 solar mass cores, cannot be a coincidence. These facts strongly suggest that the cores are in pressure equilibrium with an external source of pressure that encompasses all the cores and that physically communicates and sets their surface pressures.

Figure 6 shows the mass-radius relation for the Pipe cores. There is a relatively tight correlation between mass $(M)$ and radius $(R)$. A linear least-squares fit to the data gives $M \sim$ $R^{2.56 \pm 0.05}$. As mentioned earlier, for clouds that obey Larson's laws we would expect constant column density and $M \sim R^{2}$. However, for a core population characterized by both a constant internal thermal pressure and a constant kinetic temperature, we would expect the core volume density to also be constant, and this would result in a mass-radius relation of the form $M \sim R^{3}$. The observed relation is closer to the expectations of constant volume density than constant column density. The fact that the data points all lie clearly above the sensitivity threshold for the observations also demonstrates that the narrow range in density

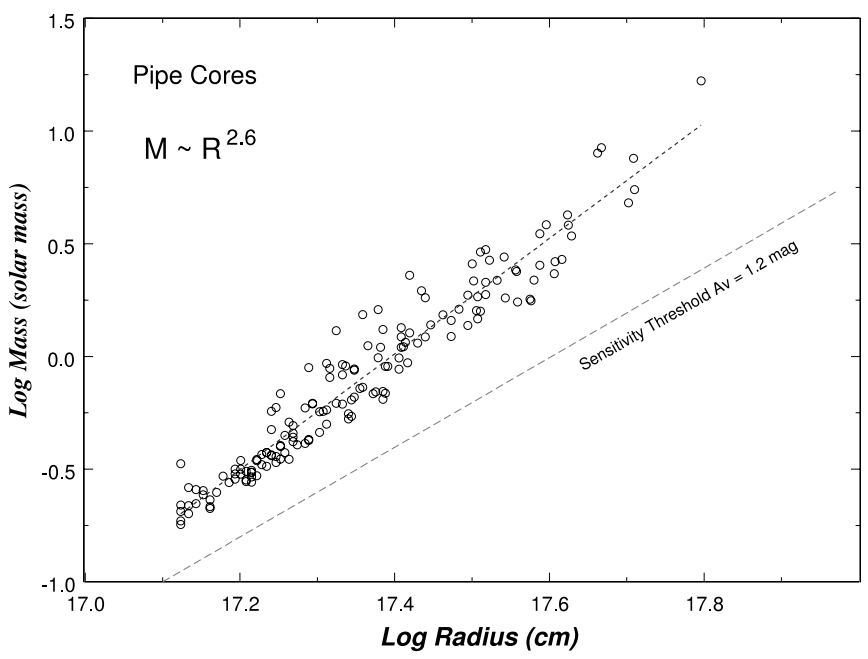

FIG. 6.-Mass-radius relation for the dense cores in the Pipe Nebula. The short-dashed line represents the least-squares fit to the data. The long-dashed line indicates the sensitivity threshold of the observations. [See the electronic edition of the Journal for a color version of this figure.] 


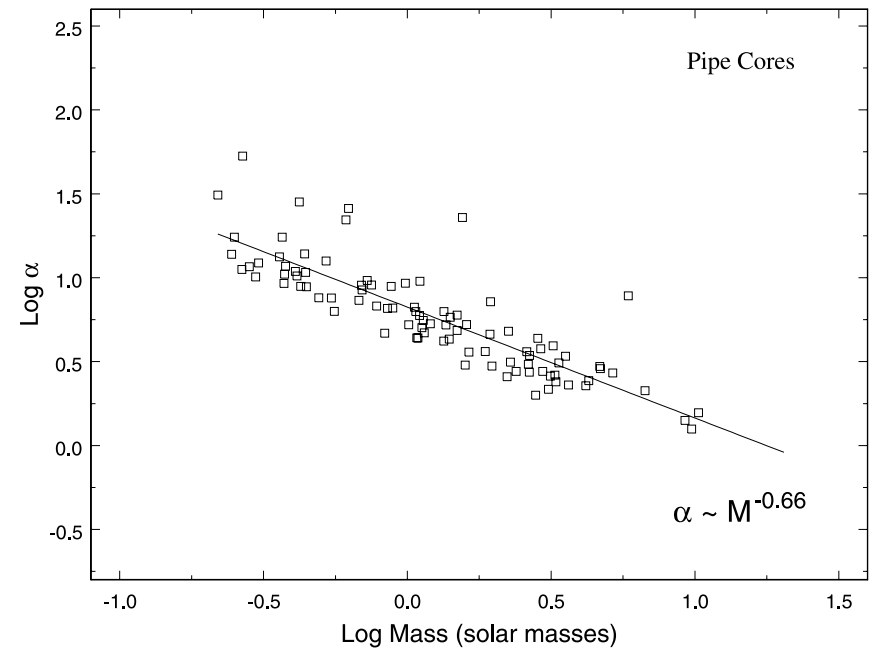

FIG. 7.- Relation between the virial parameter and core mass. The solid line is the least-squares fit to the data. [See the electronic edition of the Journal for a color version of this figure.]

derived for the cores in Figure 1 and manifest here is not an artifact of observational selection. This finding provides further evidence in support of the notion that the internal pressures of the Pipe cores are thermally dominated and are all characterized by essentially the same surface pressure.

An often used metric to evaluate the relative importance of the gravitational and kinetic energies of a dense core is the virial parameter: $\alpha=\left(5 \sigma^{2} R\right) /(G M)$ (McKee 1998). In their theoretical study of pressure confined cores in magnetized clouds, Bertoldi \& McKee (1992) argued that for pressure-confined cores $\alpha$ should depend on mass as $\alpha \sim M^{-2 / 3}$. To illustrate this consider that for an isothermal, constant density, pressure-confined core, $M \sim R^{3}$, as mentioned above, thus using the definition of $\alpha, \alpha \sim M^{1 / 3} / M \sim$ $M^{-2 / 3}$. In Figure 7 we plot $\alpha$ versus mass for the Pipe core population. There is a strong correlation between the two parameters and for all cores $\alpha>1$. A least-squares fit to the data gives $\alpha \sim M^{-0.66 \pm 0.04}$, closely matching the expectations for an ensemble of pressure-confined cores. The fact that $\alpha$ is not a constant indicates again that the dense core population is in violation of Larson's laws. The fact that none of the cores appear virialized suggests that the core population is extremely young.

These considerations imply that the cores in the Pipe are pressure confined entities. Even though most are gravitationally unbound, they are still all coherent objects that will persist as such for a significant period of time, at least one or more sound crossing times (i.e., $\tau_{\text {core }} \geq 10^{6} \mathrm{yr}$ ).

\subsubsection{The Source of the Confining Pressure}

The mean of the internal pressures of the core population is $\langle P / k\rangle=1.6 \times 10^{5} \mathrm{~K} \mathrm{~cm}^{-3}$. As mentioned earlier, this pressure corresponds to the average pressure within the cores. However, the surface pressures of the cores are likely to be lower, since most cores appear centrally concentrated and likely possess outwardly decreasing density gradients. We expect that the surface pressures of the cores are likely to be less by factors of typically 2 than their individual mean pressures calculated here, i.e, $\langle P / k\rangle_{\text {surface }} \approx 8 \times$ $10^{4} \mathrm{~K} \mathrm{~cm}^{-3}$. The estimated mean surface pressure of the cores is nearly an order of magnitude higher than the total (thermal+ turbulent) gas pressure of the interstellar medium (ISM), i.e., $P_{\mathrm{ISM}} / k \approx 10^{4} \mathrm{~K} \mathrm{~cm}^{-3}$ (Bertoldi \& McKee 1992). Thus the ISM is not likely the source of the external pressure that confines the cores. However, since the cores together represent only about $1 \%$ of the mass of the Pipe cloud, it is possible that the weight of the Pipe cloud itself is the source of the external pressure within which the cores are embedded. The pressure due to the weight of the Pipe cloud is given by

$$
P_{\text {cloud }}=(3 \pi / 20) G \Sigma^{2} \phi_{G}=4.5 \times 10^{3} \phi_{G} k A_{V}^{2},
$$

where $\Sigma$ is the mean mass surface density of the cloud i.e., $\Sigma=M_{\text {cloud }} / \pi R^{2}, k$ is Boltzmann's constant, $A_{V}$ is the corresponding mean extinction, and $\phi_{G}$ is a dimensionless correction factor to account for the nonspherical geometry of the cloud (Bertoldi $\&$ McKee 1992). Following the prescription of Bertoldi and McKee (1992), we estimate $\phi_{G}$ to be 1.6. The mean extinction we measure from the LAL06 data for the Pipe cloud corresponds to $A_{V} \approx 4$ mag. This yields $P_{\text {cloud }} / k \approx 10^{5} \mathrm{~K} \mathrm{~cm}^{-3}$. The close agreement between the estimated cloud pressure and that of the cores indicates that the cloud, itself, is likely the source of the external pressure for the cores.

We note here that turbulent ram pressure from the intercore gas could also be a significant source of external confining pressure for the cores. The turbulent ram pressure is given by $P_{\text {ram }}=$ $\rho \sigma^{2}$. For the typical observed ${ }^{13} \mathrm{CO}$ line widths of $1 \mathrm{~km} \mathrm{~s}^{-1}$ (Onishi et al. 1999) and an assumed density for ${ }^{13} \mathrm{CO}$-emitting gas of $10^{3} \mathrm{~cm}^{-3}$, we find $P_{\text {ram }} / k \approx 5 \times 10^{4} \mathrm{~K} \mathrm{~cm}^{-3}$, within a factor of 2 of the required pressure. If turbulent pressure was the confining pressure for the cores then the observed uniformity of the internal core pressures across the cloud would probably require the turbulence to be driven on large, not small scales. This is because the downward turbulent cascade from large to small scales across the cloud would more likely produce uniformity of turbulent motions on the scales of the cores than would turbulence driven more locally (e.g., by outflows). Indeed, if the pipe cloud is self-gravitating, it is quite likely that the pressure due to the weight of the cloud is transmitted and manifest by the turbulent velocity field.

Taken together the results presented in the preceding sections lead to a potentially profound implication regarding the nature of the cores in the Pipe cloud. The physical structure of a dense core is dictated by a single requirement: pressure equilibrium with a surrounding source of external pressure. The source of this external pressure is most likely the weight of the molecular cloud in which the cores were formed.

\subsubsection{On the Origin of the Apparent Variations in Core Pressures}

One of the important characteristics of the observed relation between gas pressure and core mass (Fig. 5) is the relatively large spread in the calculated mean core pressures. We briefly consider some possible causes of this spread. One of the most likely causes is a variation in the external pressure across the cloud. The cloud is certainly not uniform and variations of order a factor of 2 or so in the pressure due to local variations in the weight of the cloud or the intercore turbulence in any one region could certainly be possible. In Figure 8 we show the variation of core pressure with position in the Pipe cloud. It is clear that the cores in the "stem" of the Pipe have systematically lower pressures and dispersion in pressures than cores in the "bowl" of the Pipe. Indeed, for cores at galactic longitudes $<0$ (stem) the mean pressure is found to be $1.2 \pm 0.3 \times 10^{5} \mathrm{~K} \mathrm{~cm}^{-3}$, whereas for cores in the "bowl" the corresponding value is $1.9 \pm 1.2 \times 10^{5} \mathrm{~K} \mathrm{~cm}^{-3}$.

Another plausible source of the variation in calculated core pressures is the presence of static magnetic fields that we did not account for in the calculation of the internal core pressure. We can estimate the magnitude of field strengths needed to produce the pressure spread in Figure 5 by assuming that all the cores are 


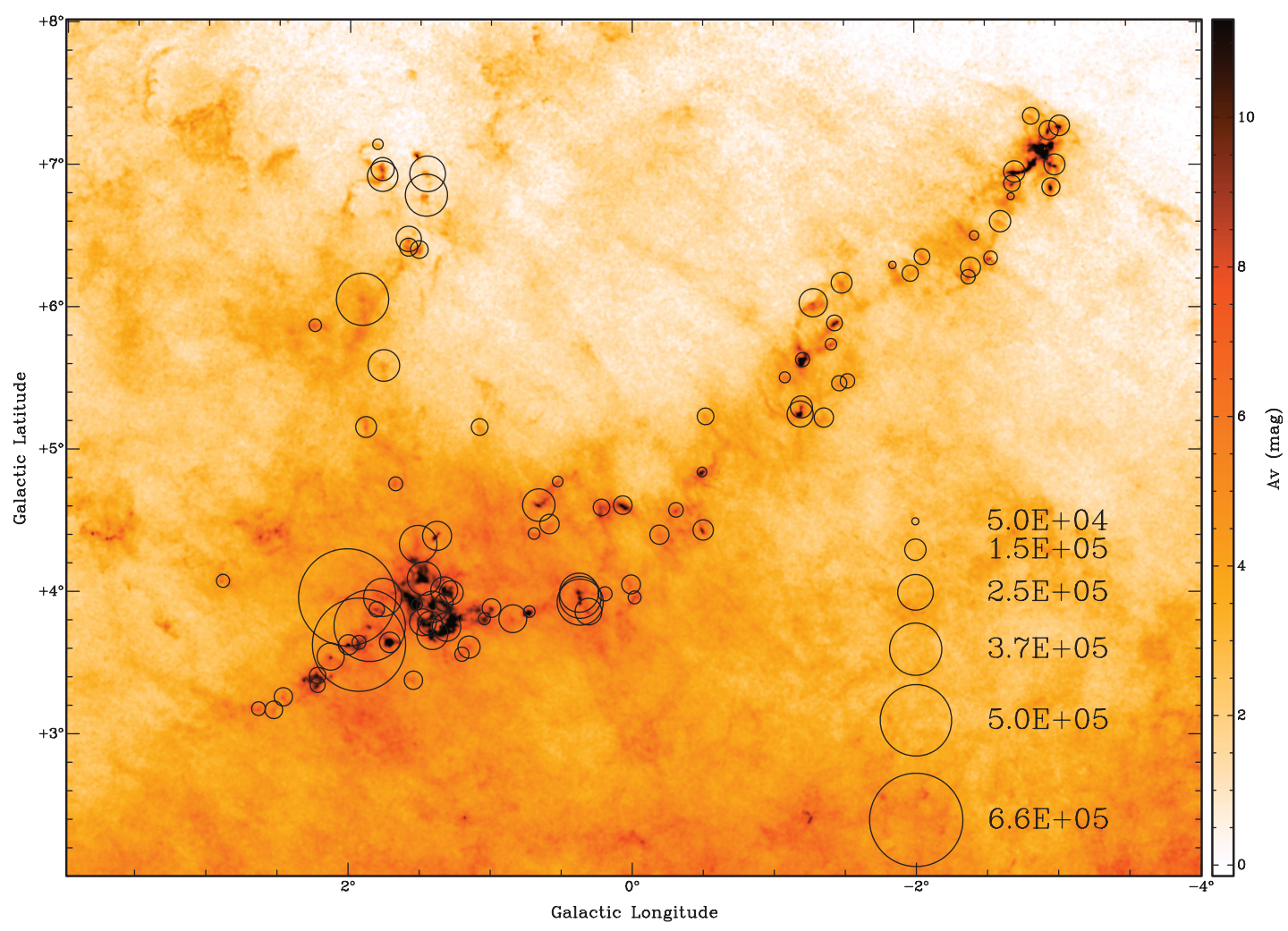

FIG. 8. - Spatial variation of internal core pressures across the Pipe cloud. The core pressures are represented by open circles whose size (area) is proportional to the total internal gas pressure in each core. These pressures are plotted on top of the near-infrared wide field extinction map of Lombardi et al. (2006).

at exactly the same pressure. We further assume that the entire variation in the calculated pressures in Figure 5 is due to a variation in the magnetic field strength within the cores. The magnetic pressure, $P_{B}$, required to bring the lowest pressure cores to the level of the constant external pressure is then $P_{B}=P_{\text {external }}-$ $P_{\text {gas }}=B^{2} / 8 \pi$, where $P_{\text {gas }}$ is the total (thermal + nonthermal) gas pressure and $B$ the strength of the static field. For the values $P_{\text {external }} / k=10^{5}$ and $P_{\text {gas }} / k=2.5 \times 10^{4} \mathrm{~K} \mathrm{~cm}^{-3}$, we find $B=$ $16 \mu \mathrm{G}$. Thus, a variation in field strength between roughly $0-16 \mu \mathrm{G}$ within the cores could produce the spread in pressures observed in Figure 5. Static field strengths of order $16 \mu \mathrm{G}$ are perhaps smaller than might be expected but certainly reasonable for these densities (Crutcher 1999).

Another possible source for the variation in calculated pressures is that our assumption of a constant gas temperature of $10 \mathrm{~K}$ in our calculation of gas pressure is not strictly correct. As mentioned earlier, $\mathrm{NH}_{3}$ observations of a sample of cores indicate that the actual gas temperatures vary between about $9.5-15 \mathrm{~K}$ with a dispersion in the measured temperatures of $\sigma\left(T_{K}\right)=2.3 \mathrm{~K}$ (Rathborne et al. 2007). However, this spread in temperature, if representative, could induce a spread of only about $25 \%$ in the actual pressures, too small to account for the bulk of the observed spread in pressure. It is also possible that our use of $\mathrm{C}^{18} \mathrm{O}$ lines to calculate the nonthermal pressure could introduce some spread in the calculated pressures since, as mentioned earlier, the pressures derived from $\mathrm{C}^{18} \mathrm{O}$ lines are probably overestimates, because the $\mathrm{C}^{18} \mathrm{O}$ lines simultaneously sample gas both in and out of the cores. This could conceivably induce a scatter of as much as a factor of 2 in some cores. However, the pressures calculated using the $\mathrm{NH}_{3}$ lines show a spread similar in magnitude to those derived from the $\mathrm{C}^{18} \mathrm{O}$ observations. This is another indication of the fact that the internal core pressures are dominated by thermal motions. Thus use of the $\mathrm{CO}$ lines to derive the nonthermal component of the total pressure is not likely a source of the variation in the calculated gas pressures.

In summary, the apparent variation in the internal core pressures is likely due to either variations in the external pressure resulting from spatial variations in the structure of the Pipe cloud, spatial variations in the intercore turbulence, or to variations in the amount of static magnetic field within the cores or to a combination of these effects.

\section{IMPLICATIONS AND DISCUSSION}

The close similarity in the shape of the Pipe CMF to that of the stellar IMF suggests a one-to-one mapping of cores to stars, modified only by a constant star formation efficiency (ALL07). Thus these cores represent the final product of the cloud fragmentation process (up to the present time). If, for example, molecular clouds can be characterized by a hierarchy of substructure produced by supersonic turbulence (Larson 1981), then these cores, with masses between $0.2-20 M_{\odot}$ and sizes of $0.1-0.4 \mathrm{pc}$ must define the physical scales for the termination of that process. Because the vast majority of these cores are starless the physical conditions that characterize them correspond to the initial conditions for star formation. In this paper we have combined previous infrared extinction and molecular-line observations to determine the basic physical properties (i.e., mass, size, density, internal pressure, etc.) and to assess the nature of this pristine population of prestellar cores.

We found the interesting result that the internal pressures of the cores are essentially dominated by thermal motions across entire spectrum of core masses from $0.2-20 M_{\odot}$. Moreover, these pressures are of similar magnitude for all the cores, independent of their mass, suggesting that the cores are in pressure equilibrium 
with their surroundings. The basic nature, structure, and subsequent evolution of these cores is thus controlled by the requirement of pressure equilibrium, self-gravity, and the fundamental thermal physics processes of heating and cooling. For example, consider that heating by cosmic rays and the interstellar radiation field coupled with cooling by molecular lines keeps the cores thermostated between $8-12 \mathrm{~K}$, a small range of temperature (e.g., Goldsmith \& Langer 1978). To maintain pressure equilibrium with their surroundings and support themselves against gravity, the cores must adjust their overall density and density structure appropriately. It is thus not accidental that their mean densities span a small range. Although most of the cores are gravitationally unbound, they are pressure confined and not transient entities. They likely evolve on acoustic timescales. It is interesting in this context that detailed molecular-line studies of the kinematics of two of the Pipe cores, B68 and FeSt 1-457, suggest that these cores are oscillating around a state of dynamical equilibrium and are thus likely to survive for at least a few sound crossing times (Lada et al. 2003, Redman et al. 2004; Keto et al. 2006; Aguti et al. 2007).

Our observations provide important constraints for understanding the origin of core masses and if there is a one-to-one mapping of the core to stellar mass, the origin of the stellar IMF as well. For example, one possible origin for the $\mathrm{CMF}$ is through gravoturbulent fragmentation. On large scales the low-density material in molecular clouds is characterized by supersonic turbulence. Indeed, the ${ }^{13} \mathrm{CO}$ emission lines from the Pipe cloud are characterized by line widths of $\approx 1 \mathrm{~km} \mathrm{~s}^{-1}$ (Onishi et al. 1999), representing bulk gas motions of about Mach 2. In this context one important constraint of our observations is the thermal and subsonic nature of the gas motions within the cores. This is in contrast to the results of numerical simulations of turbulent fragmentation that typically produce dynamic cores characterized by supersonic internal motions. In one calculation Klessen et al. (2005) found that under optimum conditions only about $25 \%$ of the cores produced by gravoturbulent fragmentation would appear to have subsonic turbulence to an observer. This is inconsistent with our observations of the Pipe cloud where $\sim 70 \%$ of the cores are characterized by subsonic turbulence (cf. Fig. 2). Another important constraint provided by our observations is the fact that the cores are pressure confined entities and in pressure equilibrium with an external pressure source, most likely provided by the weight of the Pipe cloud itself. As we discuss below, this constraint provides a potentially critical insight into the origin of the core masses and the IMF.

\subsection{Core Stability: From CMF to IMF}

As thermally dominated, dense cores in pressure equilibrium with the surrounding cloud material, the Pipe cores are perhaps most appropriately modeled as Bonnor-Ebert spheres (e.g., Johnstone et al. 2000; Alves et al. 2001). Bonnor-Ebert spheres are pressure truncated isothermal spheres in hydrostatic and pressure equilibrium with their surroundings. Bonnor (1956) and Ebert (1955) investigated the stability of such pressure-confined isothermal spheres and showed that under a specific condition such objects became unstable. This condition corresponds to the critical Bonnor-Ebert (BE) mass given by

$$
m_{\mathrm{BE}}=1.82\left(\frac{\bar{n}}{10^{4} \mathrm{~cm}^{-3}}\right)^{-0.5}\left(\frac{T}{10 \mathrm{~K}}\right)^{1.5} M_{\odot},
$$

where $\bar{n}$ is the mean volume density of the core. Above this mass cores are out of equilibrium and prone to fragmentation and/or

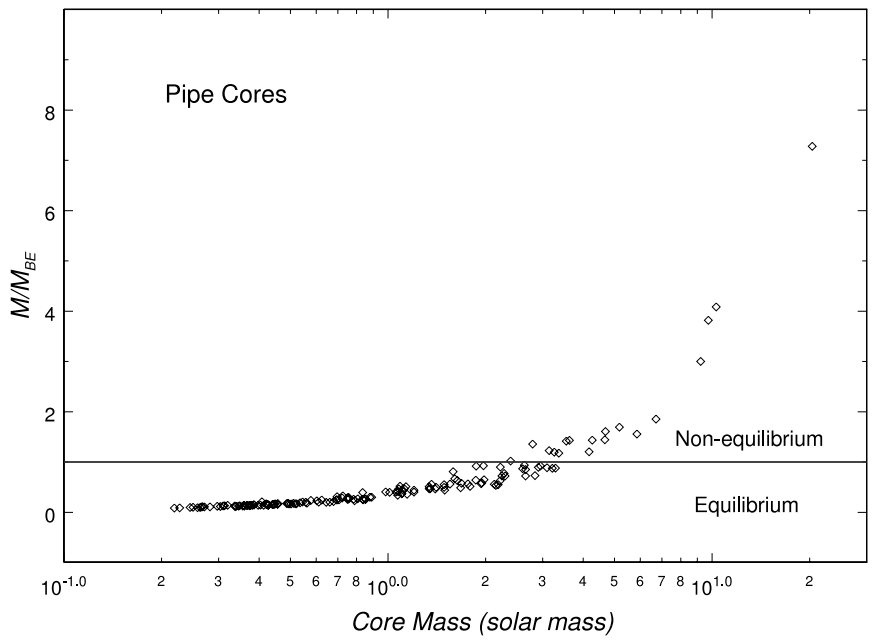

FIG. 9.- Ratio of core mass to Bonnor-Ebert critical mass for each individual core plotted against core mass. The entire core population appears to be characterized by a single critical BE mass of $\approx 2 M_{\odot}$. Cores with masses in excess of the critical mass are likely out of equilibrium and destined to form stars. There is also a large population of cores that are presently in equilibrium states. Most of these are likely in stable equilibrium states and thus are unlikely to collapse to form stars unless further perturbed via an increase in the external pressure, loss of internal pressure support (e.g., cooling), or a combination of both effects. [See the electronic edition of the Journal for a color version of this figure.]

collapse. Below this mass cores are in equilibrium states, primarily stable equilibrium states. For the mean density of cores in the Pipe cloud $\left(7.3 \times 10^{3} \mathrm{~cm}^{-3}\right)$, this critical mass is about $2 M_{\odot}$. In Figure 9 we plot the ratio of core mass to critical BE mass, $\mathrm{m} / \mathrm{m}_{\mathrm{BE}}$ (calculated individually for each core) against core mass. The two quantities form a tight relation that crosses the critical threshold $\left(m=m_{\mathrm{BE}}\right)$ at a mass of $\approx 2-3 M_{\odot}$. This is also the mass at which the cores appear to become gravitationally bound (Fig. 4).

The results presented above may have interesting ramifications for understanding the origin of the stellar IMF. In our earlier study of the Pipe CMF we showed that its overall shape was very similar to that of the stellar IMF for field stars and for the young Trapezium cluster embedded in the Orion Nebula (ALL07). However, the two functions (i.e., CMF and IMF) differed in their characteristic masses. The characteristic mass and mass scale of the $\mathrm{CMF}$ was a factor of $\approx 3$ higher than those of the stellar IMFs which were very similar to each other. This was interpreted to indicate that the stellar IMF directly originates from the CMF after modification of the individual core masses in the CMF by a uniform star formation efficiency (SFE) of $\approx 30 \%$ (ALL07). Theoretical investigations suggest that SFEs of this magnitude result from core disruption via the outflows that are generated as a natural consequence of the formation and evolution of accreting protostars (Matzner \& McKee 2000; Shu et al. 2004). In this picture each core must form one star or stellar system.

The observations in Figure 9, however, present a difficulty for such an interpretation. In order for the Pipe CMF to produce an IMF similar to that of field stars or the Trapezium Cluster, all the cores will have to form stars, yet as the figure shows, a large fraction of the cores appear to be in stable configurations. As it stands now only cores with masses in the vicinity of, or greater than, the critical BE mass will form stars. If nothing else were to happen, the IMF of the stars that would emerge from the cloud would, after adjusting for the SFE of $\sim 30 \%$, be similar to the Salpeter IMF for stellar masses greater than about $0.6-1 M_{\odot}$. Lower mass stars would also be expected to form as a result of random variations in the various important cloud paramaters, such as cloud pressure, 
internal temperature, magnetic fields, etc. (e.g., Adams \& Fatuzzo 1996). But these stars would be rare compared to stars in the 0.6-1 $M_{\odot}$ range. Indeed, it would be extremely difficult to form any brown dwarfs. It is interesting that the IMF we just described is very similar to that derived by Luhman (2004) for the Taurus cloud, a complex with similar overall properties to the Pipe. The Taurus IMF is unusual in that it does differ from the IMF of the field as well as the IMFs of young clusters such as the Trapezium and IC 348 in that it has a peak near $1 M_{\odot}$ rather than at $0.1-$ $0.3 M_{\odot}$ (Luhman 2004).

If the Pipe cores are to ultimately form an IMF more similar to the field star IMF, with a peak closer to $0.1-0.3 M_{\odot}$, then the lower mass cores must become unstable. To understand under which conditions this could happen, we rewrite the critical BE mass in terms of the external pressure, $P_{\text {ext }}$ and the sound speed, $a$ :

$$
m_{\mathrm{BE}}=1.15\left(\frac{a}{0.2 \mathrm{~km} \mathrm{~s}^{-1}}\right)^{4}\left(\frac{P_{\mathrm{ext}} / k}{10^{5} \mathrm{~K} \mathrm{~cm}^{-3}}\right)^{-0.5}
$$

To stimulate the low-mass cores to form stars we must lower the critical BE mass. The critical BE mass can be decreased in one of two possible ways. First, we can increase the external cloud pressure. To lower the BE mass to that which would result in a stellar IMF peak near $0.2 M_{\odot}$, we need to decrease the BE critical mass to $\sim 0.7 M_{\odot}$, about a factor of 3 lower. If the sound speed, $a$, is kept constant we need to increase the external pressure by about an order of magnitude to a value of $P_{\text {ext }} / k \approx 10^{6} \mathrm{~K}$ $\mathrm{cm}^{-3}$. Given that the external pressure is provided by the weight of the cloud, this would require an increase of about a factor of 3 in the cloud mass or a decrease of just under a factor of 2 in the cloud radius (eq. [2]). An increase in the total cloud mass seems unlikely, but it is not inconceivable that the cloud could gravitationally contract to nearly half its size if it somehow were to lose much of its overall support against gravity, perhaps via the dissipation of its supersonic turbulence.

A second way to decrease the critical BE mass of the cores in the Pipe Nebula would be to decrease their internal pressures by decreasing the sound speed, $a$. Because of the sensitive dependence on $a$, the BE critical mass could be significantly decreased by a small decrease in $a$. To decrease the critical BE mass by the desired factor of 3 would require only a $30 \%$ decrease in the sound speed. Since the sound speed depends on the square root of temperature, a decrease in the kinetic temperature of about a factor of 1.7 could produce the desired decrease in critical mass. For a $10 \mathrm{~K}$ core the temperature would need to cool to about $6 \mathrm{~K}$. Since heating and cooling should thermostat the cores at $\sim 10 \mathrm{~K}$, cooling below this value would be somewhat difficult. For starless cores the dust can have equilibrium temperatures as low as $5-6 \mathrm{~K}$ so if there is any degree of gas-dus coupling then the cores could be efficiently cooled by the dust to the required levels (Goldsmith 2001). How likely this is given the typical densities of the Pipe cores is difficult to assess. Loss of whatever small amount of magnetic or (subsonic) turbulent pressure the cores possess could also decrease the internal pressure and facilitate their eventual collapse. Ambipolar diffusion could naturally lead to the desired decrease in the internal magentic pressure (e.g., Adams \& Shu 2007). A combination of some cooling and loss of magnetic and turbulent pressure support is quite possible and eventually many of these low-mass cores could reach the critical mass threshold.

One potential difficulty with the above evolutionary scenario would arise if the timescale for the presently stable cores to evolve to unstable configurations is greater than a few million years.
Since the more massive unstable cores will form stars relatively quickly, the end result of the star formation process would be a stellar population displaying a mass-dependent age gradient. Although age spreads in young stellar populations can be on the order of 3-5 Myr, no evidence has yet been found for a systematic, mass dependent age gradient of similar magnitude.

It also may be difficult to increase the external pressure provided by the Pipe cloud to the extent necessary to push the lowest mass cores to collapse, form stars, and thereby produce a fully sampled, standard IMF. However, such high pressures do appear to characterize other star-forming regions, particularly those where clusters and thus most stars are formed (Lada \& Lada 2003). For example, Johnstone et al. $(2000,2001)$ have estimated $P / k \sim 10^{7}$ and $10^{6} \mathrm{~K} \mathrm{~cm}^{-3}$ for the $\rho$ Oph and Orion B cluster-forming clouds, respectively. Moreover, consider the cores of infrared dark clouds (IRDCs). These opaque, massive regions are believed to be the precursors of embedded clusters (Rathborne et al. 2006). A survey of IRDCs for millimeter-wave emission from dust provides mass and size estimates for 140 cold dense cores within IRDCs (Rathborne et al. 2006). The median mass and radius for this sample of cores are $121 M_{\odot}$ and $0.27 \mathrm{pc}$, respectively. The corresponding pressure from equation (2) is $P_{\text {ext }} / k=2.7 \times 10^{6} \mathrm{~K} \mathrm{~cm}^{-3}$. For such levels of pressure the corresponding critical BE mass could decrease substantially. However, this is only true if the sound speed, $a$, remained similar to that in the Pipe cloud. It is more likely that in such compact massive regions the sound speed in equation (4) is higher than that of a $10 \mathrm{~K}$ purely thermal gas. Given the sensitivity of the BE critical mass to $a$, this could easily compensate for the increase in external pressure, leaving the critical BE mass similar to that in the Pipe. Indeed, this appears to be the case for the cores in the Ophiuchus and Orion B clouds studied by Johnstone et al. (2000, 2001). For the Ophiuchus cores the typical kinetic temperature was estimated to be $\sim 20 \mathrm{~K}$ and the corrresponding surface pressure $P / k \sim 3 \times 10^{6}$, comparable to the external pressure exerted by the cloud itself, similar to the situation for the Pipe. The critical BE mass in that cloud was found to be $\sim 1-2 M_{\odot}$ with most of the cores being subcritical, again similar to the situation in the Pipe. For the cores in Orion B the typical kinetic temperature was found to be $\sim 30 \mathrm{~K}$ with the corresponding surface pressure $\sim 10^{6} \mathrm{~K} \mathrm{~cm}^{-3}$ and the critical BE mass $\sim 3 M_{\odot}$. However, given the larger values for $a$ and internal temperatures in such high-pressure regions, cores with masses below the critical BE mass may have more room to cool and lose internal pressure support than similar cores in lower pressure regions like the Pipe. Thus it may be easier to produce low-mass and even substellar objects in clustered environments than in regions similar to the Pipe or Taurus clouds. Because the formation of brown dwarfs does require restrictive conditions, one might expect the substellar portion of the IMF in a recently formed stellar population to be very sensitive to local conditions and thus to vary noticeably from region to region.

In the preceeding discussion of the possible evolution of the cores in the Pipe CMF, it was assumed that the core masses were fixed and unchanging. Another possibility worthy of consideration is that the core population in the Pipe is so young that the cores have not yet obtained their final masses. This, in particular, could have important consequences for the evolution of the presently stable low-mass cores in the cloud. Separate studies by Clark and Bonnell (2005) and Gomez et al. (2007) have raised the possibility that dense cores formed from a turbulent medium could grow in mass with time, starting out as unbound low-mass objects and ending up as bound objects at the threshold of collapse and fragmentation. In this picture the stable but unbound cores in the Pipe would be still gaining mass and as a result will 
eventually cross the critical BE threshold and ultimately collapse to form stars. The resulting IMF would not bear any relation to the original CMF. Whether such models can account for the basic physical properties of the cores reported here remains to be determined.

\subsection{The Origin of the CMF: Thermal Fragmentation Under Pressure}

The fact that the critical BE threshold for the entire core population corresponds to a mass of $\sim 2 M_{\odot}$, as shown by Figure 9, is potentially very significant. This becomes apparent when one considers the CMF of the Pipe cloud derived from the extinction observations by ALL07. These authors found the CMF to rise, with decreasing mass, in a power-law fashion, from the highest mass $\left(\sim 20 M_{\odot}\right)$ core to roughly $2-3 M_{\odot}$ (similar to the behavior of the Salpeter or stellar IMF). At this mass the CMF was found to break or depart from the power law. The CMF then continued to rise only very slowly, forming a broad peak between $2-0.4 M_{\odot}$, before declining toward lower masses (cf. Fig. 3 of ALL07). The $\mathrm{CMF}$ indicates that most of the cores that formed in the Pipe cloud formed with masses between $0.4-2.0 M_{\odot}$. The departure point from the Salpeter-like power law sets a characteristic mass of $\sim 2-3 M_{\odot}$ for the CMF in this cloud. The fact that this characteristic mass is very close to the critical BE mass for the cores is unlikely to be a chance coincidence; indeed, it provides a potentially interesting clue concerning the very origin of the CMF. The physical interpretation of this result is straightforward: the characteristic mass of the CMF (and the CMF itself) is the direct result of thermal fragmentation in a pressurized medium. In other words, the CMF may have its origin in the physical process of pressurized thermal fragmentation.

It is instructive in this context to express the core mass function, $\mathrm{CMF}(\log m)$, in terms of the characteristic mass, $m_{c}$, a shape parameter, $s_{i}$ and an arbitrary constant, $c_{0}$, as follows:

$$
\mathrm{CMF}(\log m)=c_{0} \Psi\left(\log \left(m / m_{c}\right), s_{i}\right)
$$

The results of this paper suggest that $m_{c}=m_{\mathrm{BE}}$. If the functional form of the stellar IMF is the same as that of the CMF but with a characteristic mass reduced by the SFE then we can express the IMF as follows:

$$
\operatorname{IMF}(\log m)=c_{1} \Psi\left(\log \left(m / m_{c}^{*}\right), s_{i}\right)
$$

with

$$
m_{c}^{*}=m_{\mathrm{BE}} \mathrm{SFE} .
$$

Expressed in this way these two equations suggest a possible generalization of our results. If we assume that the shape of the CMF is invariant, that is, $s_{i}$ is constant, then the CMF is completely specified by one parameter, the critical BE mass, which in turn depends on only two simple physical parameters, external pressure, $P_{\text {ext }}$, and sound speed, $a$ (i.e., eq. [4]). This has the powerful implication that knowledge of the pressure and sound speed could be used to predict the CMF (and ultimately the IMF) that would be produced in any given star-forming environment, near or far, past or present. In principle, both parameters $a$ and $P_{\text {ext }}$, can be directly obtained or inferred from observations.

Whether our conjecture of an invariant shape for the CMF is a realistic one, however, remains to be verified by both observa- tion and theory. This conjecture can be directly tested observationally with additional extinction and dust emission surveys of dense gas in other molecular cloud complexes. Indeed, numerous recent determinations of the CMFs using observations of dust emission from cores in a number of other clouds certainly appear to support our conjecture (e.g., Motte et al. 2001; Testi \& Sargent 1998; Johnstone et al. 2000, 2001; Beuther \& Schilke 2004; Enoch et al. 2006; Stanke et al. 2006; Walsh et al. 2007). Moreover, if the stellar IMF originates directly from a one-toone transformation of the CMF, then the observed similarity of the IMFs in young clusters with that of the field perhaps also points to an invariant CMF shape function. In addition, theoretical considerations have suggested that the CMF (and IMF) could be characterized by a simple lognormal form or shape. This is a natural outcome of the central limit theorem if a number of independent physical variables contribute to the final determination of initial core masses (e.g., Adams \& Fatuzzo 1996). On the one hand, this has to some extent been borne out by numerical simulations of gravitational fragmentation (e.g., Klessen et al. 1998). On the other hand, simulations of turbulent fragmentation do not produce a universal CMF (Ballesteros-Paredes et al. 2006). Although recent observations support the conjecture of a universal shape for the CMF, the theoretical situation is unclear.

\subsection{Speculations on the Origin of Stellar Multiplicity}

Stellar multiplicity is a fundamental parameter of stellar systems. It is an increasing function of stellar mass. The single star fraction (SSF) for M stars $\left(m \sim 0.1-0.6 M_{\odot}\right)$ is measured to be around $70 \%$ and since the vast majority of stars formed are $\mathrm{M}$ stars, most stars produced in the Galaxy are single (Lada 2006). The SSF declines steadily along the Salpeter, power-law portion of the IMF with the most massive (OB) stars being characterized by a SSF $\leq 20 \%$ (i.e., corresponding to the highest multiplicity fraction). Existing theoretical attempts to account for stellar multiplicity statistics (e.g., Durisen et al. 2001; Kroupa 1995) are unable to simultaneously explain both the mass dependence and the overall magnitude of the SSF. For the Pipe cloud the peak of the IMF nearly coincides with the critical BE mass. It is difficult to imagine how critical mass, thermally supported, hydrostatic cores in pressure equilibrium with their surroundings could fragment any further to form binary or multiple star systems. Such cores are more likely to directly collapse in an inside-out fashion to form a single star (Shu 1977; Shu et al. 1987; Foster \& Chevalier 1993). However, cores whose masses exceed the critical mass are not only highly unstable but also out of equilibrium, having masses ranging from just above $m_{\mathrm{BE}}$ to $\approx 10 m_{\mathrm{BE}}$ (Fig. 9). Numerical simulations of the collapse and fragmentation of isothermal clouds whose masses exceed the Jeans mass suggest that the number of fragments formed in such a process is comparable to the initial number of Jeans masses in the cloud with the result that binary systems and hierarchical multiple systems are frequently produced (Larson 1978; Boss \& Bodenheimer 1979). Thus cores with masses in excess of the critical BE mass should be increasingly susceptible (with increasing mass) to fragmentation and multiple star formation. The outcome of star formation in these cores might thus be expected to produce an increasing multiplicity fraction with mass along the Salpeter, power-law portion of the IMF, similar to what is observed. In this context it also is interesting to note that B59, the most massive core in the Pipe (and the only one so far known to form stars), has a measured mass of $\sim 20 M_{\odot}\left(\sim 10 m_{\mathrm{BE}}\right)$ and has already fragmented and produced a group of $\sim 13$ low-mass young stars (Brooke et al. 
2007). ${ }^{7}$ For the most part cores with masses less than the critical mass are presently stable against collapse. However, if these cores experience either a slow increase of external pressure or a gradual decrease in internal pressure or some combination of both effects, they will cross the equilibrium threshold at just the critical $\mathrm{BE}$ mass. Thus they are also likely to produce single stars. Since these objects and the presently critically stable objects represent the peak of the CMF and the bulk of the cores formed, we expect most stars formed in the Pipe to be single, similar to what is observed for field stars (Lada 2006). If the CMF of the Pipe is the result of thermal fragmentation in a pressurized medium, and is transformed directly into the IMF by the SFE, then the overall magnitude and mass dependence of stellar multiplicity may be a natural outcome of this straightforward core fragmentation process.

\section{SUMMARY AND CONCLUSIONS}

We have combined previous infrared extinction and millimeterwave molecular-line observations to determine the physical nature of the population of dense cores in a single molecular cloud, the Pipe Nebula. We summarize the primary results of the paper as follows:

1. The cores are found to be relatively dense objects that display a narrow range in number density with a median value of $n=7.1 \pm 2.1 \times 10^{3} \mathrm{~cm}^{-3}$.

2. Widths of $\mathrm{C}^{18} \mathrm{O}$ and $\mathrm{NH}_{3}$ lines observed toward the cores are not correlated with core size or mass and do not obey a linewidth-size relation. The nonthermal velocity dispersions measured in both tracers are also independent of core size and mass and are predominately characterized by subsonic $(70 \%)$ or transsonic $(25 \%)$ motions.

3 . The ratio, $R_{p}$, of thermal to nonthermal gas pressure is found to range between roughly 0.2 and 100 and thermal pressure is found to exceed nonthermal (turbulent) gas pressure in the large majority $(\sim 67 \%-80 \%)$ of the cores. Thermal pressure support is significant (i.e., $\left.R_{p}>0.5\right)$ for nearly all $(\sim 90 \%)$ the cores.

4. The core internal pressures are surprisingly similar over the entire $0.2-20 M_{\odot}$ range of core mass and exceed the expected total gas pressure of the ISM by nearly an order of magnitude. The similarity of their internal pressures indicates that the cores are in pressure equilibrium with an external pressure source. The source of this external pressure is likely provided by the overall weight of the Pipe cloud in which the cores are embedded.

5. The dispersion in internal core pressure of about a factor of $2-3$ is significant and likely results from either local variations in the external pressure due to structural variations in the Pipe cloud or the presence of internal static magnetic fields with strengths between $0-16 \mu \mathrm{G}$ or a combination of both.
6. Only the most massive $\left(m \geq 2 M_{\odot}\right)$ cores are gravitationally bound. Although the majority of cores are gravitationally unbound they appear to be pressure-confined, coherent objects.

7. The entire core population is found to be characterized by the same critical Bonnor-Ebert mass of $\sim 2 M_{\odot}$. This mass is very similar to the observed characteristic mass of the CMF in this cloud (ALL07). This in turn may suggest that the CMF, the direct progenitor of the stellar IMF, originates as a result of thermal fragmentation in a pressurized medium.

This last conclusion is potentially very significant because it suggests that the CMF that is produced out of a molecular cloud may be able to be specified by only a few basic physical parameters, such as external pressure and temperature, to name two. Moreover, the structure and evolution of dense cores may depend on the interplay of only a small and restricted set of basic physical parameters/processes such as self-gravity, heating, and cooling of the core gas and the pressure of the external medium.

Another key finding of our study is that the core formation process simultaneously produced many objects that are in apparently stable configurations in addition to a number of bound, nonequilibrium objects. The presently stable cores are unlikely to undergo star formation unless they experience further evolution driven by either an increase in external pressure or a decrease in their internal pressures or a combination of both. If only those cores that are presently bound, and either out of equilibrium or critically stable, collapse to form stars, the stellar IMF that will emerge from this cloud will resemble that of the Taurus star-forming region, not that of the field or of embedded young clusters where most stars are formed. It would also appear very difficult to form substellar objects from a set of such pressure confined cores. The formation of lower mass stars, brown dwarfs and a standard IMF may be facilitated in regions characterized by higher external pressures, such as massive cluster-forming cores.

Since most cores produced in a pressurized thermal fragmentation process appear to have masses near the critically stable value, the stars that form from them would tend to be single. The observed increase in stellar multiplicity with mass along the Salpeter portion of the stellar IMF may be a natural consequence of the additional production of cores in this type of fragmentation process that have masses increasingly in excess of the critical value and thus increasingly likely to form multiple stellar systems.

Finally, the most important result of our study may be the recognition of the significant role played by pressure in determining both the physical natures of dense cores and the process that leads to their formation and as a result to the development of the dense core and initial stellar mass functions.

\footnotetext{
${ }^{7}$ The production of increasing multiplicity with core mass along the Salpeter portion of the CMF suggests that a departure from a strict one-to-one mapping of the CMF to the IMF may occur for the more massive cores. The overall similarity of the CMF to the IMF over most of this regime would seem to indicate that cores in the $2-10 M_{\odot}$ range produce binary and multiple systems in which the primary stars dominate the masses of the stellar systems. However, for the most massive cores (e.g., B59) that produce stellar clusters, the concept of a strict one-to-one mapping between the two mass functions is not likely to remain valid.
}

We are indebted to Frank Shu, Ramesh Narayan, and Doug Johnstone for enlightening discussions. We thank the referee Ian Bonnell for criticisms and suggestions that improved the paper. This research was supported in part by NASA Origins grant NAG-13041.

\section{APPENDIX}

The basic physical parameters of the Pipe extinction cores calculated from the infrared extinction observations of Lombardi et al. (2006) are listed in the Table 1. These respectively include the core identification number (ID), mass, radius and density. The mass is the background-subtracted mass derived from the wavelet decomposition of the infrared extinction map by Alves et al. (2007) assuming a standard gas-to-dust abundance. 
TABLE 1

Physical Core Properties

\begin{tabular}{|c|c|c|c|}
\hline Pipe Core ID & $\begin{array}{l}\text { Mass } \\
\left(M_{\odot}\right)\end{array}$ & $\begin{array}{l}\text { Radius } \\
\left(\mathrm{cm}\left[\times 10^{17}\right]\right)\end{array}$ & $\begin{array}{c}\text { Density } \\
\left(\mathrm{cm}^{-3}\left[\times 10^{4}\right]\right)\end{array}$ \\
\hline 1 & 0.38 & 1.85 & 0.73 \\
\hline 2 & 0.46 & 2.04 & 0.66 \\
\hline 3.1. & 0.49 & 2.12 & 0.63 \\
\hline 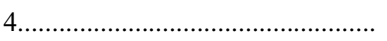 & 0.36 & 1.85 & 0.69 \\
\hline 5 & 0.23 & 1.50 & 0.82 \\
\hline 6 & 3.14 & 3.57 & 0.84 \\
\hline 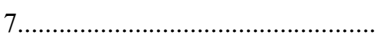 & 4.69 & 4.45 & 0.65 \\
\hline 8 & 3.26 & 3.76 & 0.75 \\
\hline 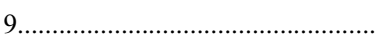 & 0.56 & 2.27 & 0.59 \\
\hline 10 & 0.51 & 2.09 & 0.68 \\
\hline 11 . & 3.37 & 3.93 & 0.68 \\
\hline 12 & 20.37 & 7.06 & 0.71 \\
\hline 13 & 0.54 & 2.04 & 0.78 \\
\hline 14 & 9.73 & 5.19 & 0.85 \\
\hline 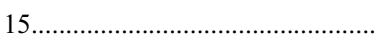 & 2.64 & 3.58 & 0.70 \\
\hline 16 & 3.29 & 4.66 & 0.40 \\
\hline $17 \ldots$ & 0.69 & 2.27 & 0.72 \\
\hline 18 & 0.35 & 1.82 & 0.70 \\
\hline 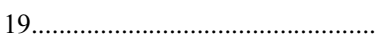 & 0.34 & 1.73 & 0.79 \\
\hline 20 & 2.28 & 3.53 & 0.64 \\
\hline $21 \ldots$ & 2.66 & 4.29 & 0.41 \\
\hline 22 & 1.01 & 2.42 & 0.86 \\
\hline 23 & 1.87 & 3.27 & 0.65 \\
\hline 24 & 0.70 & 2.29 & 0.71 \\
\hline $25 \ldots$ & 1.10 & 2.78 & 0.63 \\
\hline $26 \ldots$ & 0.37 & 1.85 & 0.72 \\
\hline 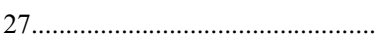 & 3.09 & 4.37 & 0.45 \\
\hline 28 & 0.32 & 1.54 & 1.07 \\
\hline 29 & 0.43 & 2.07 & 0.59 \\
\hline 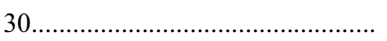 & 0.41 & 1.99 & 0.64 \\
\hline 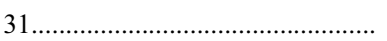 & 1.95 & 3.61 & 0.50 \\
\hline 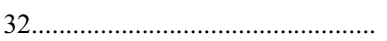 & 0.45 & 1.97 & 0.72 \\
\hline 33... & 4.27 & 4.37 & 0.62 \\
\hline 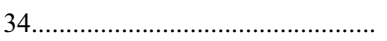 & 2.66 & 3.85 & 0.57 \\
\hline 35 & 0.52 & 2.19 & 0.60 \\
\hline 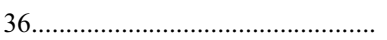 & 1.69 & 3.16 & 0.65 \\
\hline 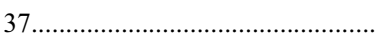 & 1.97 & 2.70 & 1.22 \\
\hline 38. & 1.10 & 2.75 & 0.64 \\
\hline 39, & 1.07 & 2.51 & 0.83 \\
\hline 40, & 9.23 & 5.77 & 0.59 \\
\hline $41 \ldots$ & 1.08 & 2.34 & 1.03 \\
\hline 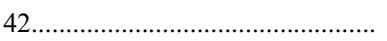 & 2.79 & 2.96 & 1.31 \\
\hline 43 & 0.85 & 2.74 & 0.51 \\
\hline 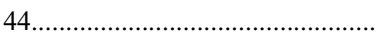 & 0.50 & 2.17 & 0.60 \\
\hline 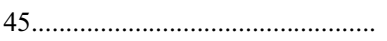 & 0.64 & 2.47 & 0.52 \\
\hline 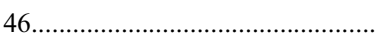 & 0.28 & 1.64 & 0.79 \\
\hline 47 & 1.41 & 2.93 & 0.68 \\
\hline 48 & 4.18 & 4.80 & 0.46 \\
\hline 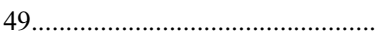 & 0.85 & 2.68 & 0.54 \\
\hline 50 & 0.40 & 1.94 & 0.67 \\
\hline $51 \ldots$ & 1.20 & 2.87 & 0.62 \\
\hline 52 & 0.24 & 1.54 & 0.82 \\
\hline 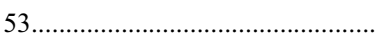 & 2.13 & 4.09 & 0.38 \\
\hline 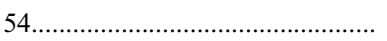 & 1.40 & 3.03 & 0.61 \\
\hline 55 & 0.30 & 1.67 & 0.80 \\
\hline 56 & 5.18 & 4.74 & 0.59 \\
\hline 57 & 0.31 & 1.60 & 0.92 \\
\hline 58 & 0.79 & 2.74 & 0.47 \\
\hline 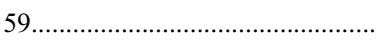 & 0.37 & 1.85 & 0.72 \\
\hline 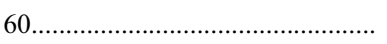 & 0.43 & 2.02 & 0.63 \\
\hline 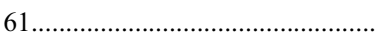 & 2.60 & 3.72 & 0.62 \\
\hline 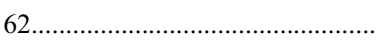 & 2.30 & 3.72 & 0.55 \\
\hline 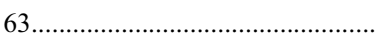 & 0.36 & 1.70 & 0.89 \\
\hline 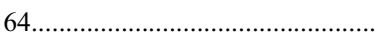 & 0.41 & 1.50 & 1.47 \\
\hline
\end{tabular}


TABLE $1-$ Continued

\begin{tabular}{|c|c|c|c|}
\hline Pipe Core ID & $\begin{array}{l}\text { Mass } \\
\left(M_{\odot}\right)\end{array}$ & $\begin{array}{c}\text { Radius } \\
\left(\mathrm{cm}\left[\times 10^{17}\right]\right)\end{array}$ & $\begin{array}{c}\text { Density } \\
\left(\mathrm{cm}^{-3}\left[\times 10^{4}\right]\right)\end{array}$ \\
\hline 65 & 0.72 & 1.99 & 1.12 \\
\hline 66 & 0.98 & 2.34 & 0.94 \\
\hline 67 & 2.84 & 4.56 & 0.37 \\
\hline 68 & 0.38 & 1.82 & 0.76 \\
\hline 69 & 1.98 & 3.43 & 0.60 \\
\hline 70 & 1.14 & 2.31 & 1.12 \\
\hline $71 \ldots$ & 0.42 & 1.79 & 0.89 \\
\hline 72 & 0.72 & 2.17 & 0.86 \\
\hline 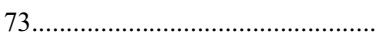 & 0.68 & 2.47 & 0.55 \\
\hline 74 & 2.96 & 4.06 & 0.54 \\
\hline 75 & 0.25 & 1.50 & 0.90 \\
\hline 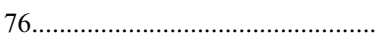 & 0.52 & 2.19 & 0.60 \\
\hline 77 & 0.40 & 1.91 & 0.71 \\
\hline 78. & 0.34 & 1.85 & 0.65 \\
\hline 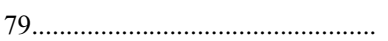 & 1.49 & 3.10 & 0.61 \\
\hline 80 & 3.21 & 4.57 & 0.41 \\
\hline 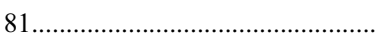 & 0.43 & 1.88 & 0.78 \\
\hline $82 \ldots \ldots \ldots \ldots$ & 0.44 & 1.99 & 0.68 \\
\hline $83 \ldots \ldots \ldots$ & 0.76 & 2.38 & 0.68 \\
\hline 84 & 0.49 & 2.02 & 0.73 \\
\hline 85 & 0.66 & 2.49 & 0.52 \\
\hline 86. & 1.12 & 2.42 & 0.96 \\
\hline 87 & 10.29 & 5.24 & 0.87 \\
\hline 88 & 2.25 & 3.63 & 0.57 \\
\hline 89 & 1.36 & 2.62 & 0.93 \\
\hline 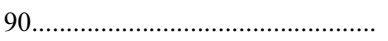 & 0.49 & 2.02 & 0.72 \\
\hline 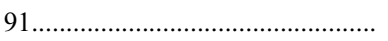 & 1.09 & 2.19 & 1.26 \\
\hline 92 & 1.61 & 2.74 & 0.95 \\
\hline 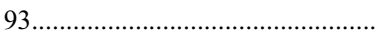 & 3.55 & 3.66 & 0.88 \\
\hline 94 & 1.06 & 2.51 & 0.82 \\
\hline 95 & 0.70 & 1.97 & 1.12 \\
\hline 96 & 1.11 & 2.45 & 0.92 \\
\hline 97 & 5.86 & 5.68 & 0.39 \\
\hline 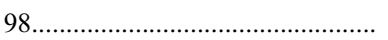 & 1.34 & 2.72 & 0.81 \\
\hline 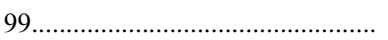 & 2.22 & 3.10 & 0.91 \\
\hline 100 & 0.61 & 2.31 & 0.60 \\
\hline $101 \ldots$ & 1.87 & 2.58 & 1.34 \\
\hline $102 \ldots \ldots$ & 6.71 & 5.79 & 0.42 \\
\hline $103 \ldots \ldots$ & 0.27 & 1.54 & 0.89 \\
\hline $104 \ldots \ldots$ & 0.53 & 2.09 & 0.71 \\
\hline $105 \ldots \ldots \ldots$ & 1.64 & 2.89 & 0.83 \\
\hline 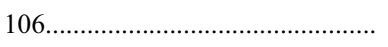 & 0.83 & 2.02 & 1.24 \\
\hline $107 \ldots$ & 0.46 & 1.94 & 0.77 \\
\hline 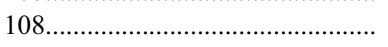 & 0.78 & 2.49 & 0.62 \\
\hline 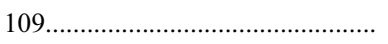 & 3.63 & 3.72 & 0.86 \\
\hline 110 & 0.37 & 1.76 & 0.82 \\
\hline 111 & 0.22 & 1.50 & 0.79 \\
\hline 112 & 1.59 & 2.38 & 1.43 \\
\hline 113 & 2.39 & 3.07 & 1.01 \\
\hline 114 & 1.14 & 2.95 & 0.55 \\
\hline 115 & 0.89 & 2.58 & 0.64 \\
\hline 116 & 1.20 & 2.70 & 0.75 \\
\hline 117 & 0.58 & 1.97 & 0.93 \\
\hline 118 & 0.62 & 2.07 & 0.86 \\
\hline 119 & 0.88 & 2.56 & 0.64 \\
\hline 120 & 0.42 & 1.88 & 0.77 \\
\hline $121 \ldots$ & 2.15 & 4.25 & 0.34 \\
\hline 122 & 1.34 & 2.89 & 0.68 \\
\hline 123 & 1.55 & 2.96 & 0.73 \\
\hline 124 & 0.34 & 1.82 & 0.69 \\
\hline $125 \ldots$ & 0.26 & 1.64 & 0.72 \\
\hline 126 & 1.50 & 3.35 & 0.48 \\
\hline $127 \ldots \ldots$ & 1.49 & 2.89 & 0.75 \\
\hline 128 & 0.27 & 1.50 & 0.96 \\
\hline 129 & 0.36 & 1.88 & 0.66 \\
\hline
\end{tabular}


TABLE $1-$ Continued

\begin{tabular}{|c|c|c|c|}
\hline Pipe Core ID & $\begin{array}{l}\text { Mass } \\
\left(M_{\odot}\right)\end{array}$ & $\begin{array}{c}\text { Radius } \\
\left(\mathrm{cm}\left[\times 10^{17}\right]\right)\end{array}$ & $\begin{array}{c}\text { Density } \\
\left(\mathrm{cm}^{-3}\left[\times 10^{4}\right]\right)\end{array}$ \\
\hline $130 \ldots \ldots \ldots$ & 0.75 & 2.22 & 0.84 \\
\hline 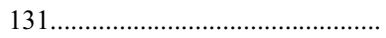 & 2.91 & 4.07 & 0.53 \\
\hline 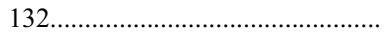 & 4.67 & 4.76 & 0.53 \\
\hline $133 \ldots \ldots \ldots . .$. & 1.94 & 3.66 & 0.48 \\
\hline 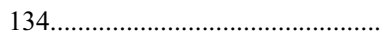 & 2.19 & 4.24 & 0.35 \\
\hline $135 \ldots \ldots \ldots$ & 0.44 & 1.97 & 0.71 \\
\hline 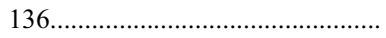 & 1.79 & 3.63 & 0.46 \\
\hline $137 \ldots \ldots \ldots \ldots \ldots \ldots$ & 0.30 & 1.60 & 0.88 \\
\hline 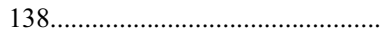 & 0.26 & 1.64 & 0.73 \\
\hline 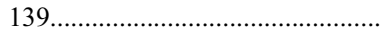 & 1.68 & 3.53 & 0.47 \\
\hline $140 \ldots \ldots \ldots$ & 1.07 & 2.87 & 0.55 \\
\hline $141 \ldots \ldots \ldots$ & 0.75 & 2.42 & 0.64 \\
\hline $142 \ldots \ldots \ldots$ & 0.39 & 1.79 & 0.82 \\
\hline 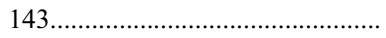 & 0.35 & 1.76 & 0.78 \\
\hline 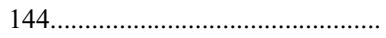 & 0.31 & 1.57 & 0.98 \\
\hline 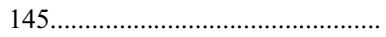 & 0.56 & 2.09 & 0.74 \\
\hline 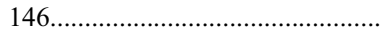 & 0.71 & 2.31 & 0.69 \\
\hline 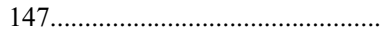 & 0.37 & 1.79 & 0.78 \\
\hline 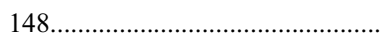 & 0.45 & 1.91 & 0.79 \\
\hline 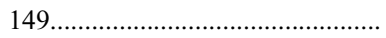 & 0.39 & 1.76 & 0.86 \\
\hline 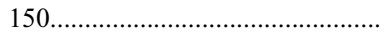 & 0.81 & 2.51 & 0.62 \\
\hline 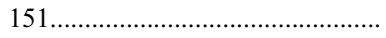 & 1.35 & 2.91 & 0.67 \\
\hline 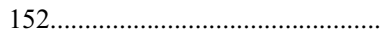 & 0.46 & 1.94 & 0.77 \\
\hline 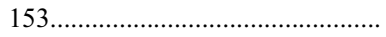 & 0.83 & 2.66 & 0.54 \\
\hline 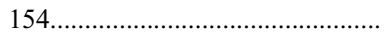 & 0.60 & 2.09 & 0.80 \\
\hline 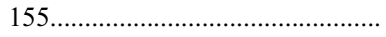 & 2.22 & 3.94 & 0.44 \\
\hline 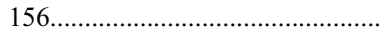 & 0.27 & 1.57 & 0.85 \\
\hline 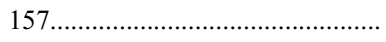 & 0.76 & 2.22 & 0.84 \\
\hline 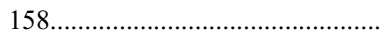 & 1.76 & 3.35 & 0.57 \\
\hline 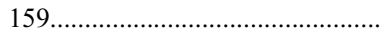 & 0.84 & 2.75 & 0.49 \\
\hline
\end{tabular}

\section{REFERENCES}

Adams, F. C., \& Fatuzzo, M. 1996, ApJ, 464, 256

Adams, F. C., \& Shu, F. 2007, ApJ, 671, 497

Aguti, E., Lada, C. J., Alves, J. F., Bergin, E. A., \& Birkinshaw, M. 2007, ApJ, 655,457

Alves, J. F., Lada, C. J., \& Lada, E. A. 2001, Nature, 409, 159

Alves, J. F., Lombardi, M., \& Lada, C. J. 2007, A\&A, 462, L17 (ALL07)

Ballesteros-Paredes et al. 2006, ApJ, 637, 384

Barranco, J. A., \& Goodman, A. A. 1998, ApJ, 504, 207

Bertoldi, F., \& McKee. C. F. 1992, ApJ, 395, 140

Beuther, H., \& Schilke, P. 2004, Science, 303, 1167

Bonnell, I. A., Larson, R. B., \& Zinnecker, H. 2007, in Protostars \& Planets V, ed. B. Reipurth, D. Jewitt \& K. Keil (Tucson: Univ. Arizona Press), 149

Bonner, W. 1956, MNRAS, 116, 351

Boss, A. P., \& Bodenheimer, P. 1979, ApJ, 234, 289

Brooke, T. Y., et al. 2007, ApJ, 655, 364

Clark, P. C., \& Bonnell, I. A. 2005, MNRAS, 361, 2

Corbelli, E., Palla, F., \& Zinnecker, H. 2005, The Initial Mass Function 50 Years Later (Dordrecht: Springer)

Crutcher, R. M. 1999, ApJ, 520, 706

Durisen, R. H., Sterzik, M. F., \& Pickett, B. K. 2001, A\&A, 371, 952

Ebert, R. 1955, Z. Astrophys. 37, 217

Enoch, M. L., et al. 2006, ApJ, 638, 293

Foster, P. N., \& Chevalier, R. A. 1993, ApJ, 416, 303

Goldsmith, P. F. 2001, ApJ, 557, 736

Goldsmith, P. F., \& Langer, W. D. 1978, ApJ, 222, 881

Gomez, G. C., Vazquez-Semadeni, E., Shadmehri, M., Ballesteros-Parades, J. 2007, ApJ, 669, 1042

Goodman, A. A., Barranco, J. A., Wilner, D. J., \& Heyer, M. H. 1998, ApJ, 504,223

Jijina, J., Myers, P. C., \& Adams, F. C. 1999, ApJS, 125, 161

Johnstone, D., Fich, M., Mitchell, G. F., \& Moriarty-Schieven, G. 2001, ApJ, 559,307

Johnstone, D., Wilson, C. D., Moriarty-Schieven, G., Joncas, G., Smith, G., Gregersen, E., \& Fich, M. 2000, ApJ, 545, 327
Keto, E., Broderick, A. E., Lada, C. J., \& Narayan, R. 2006, ApJ, 652, 1366 Klessen, R. S., Ballesteros-Paredes, J., Vázquez-Semadeni, E., \& Durán-Rojas, C. 2005, ApJ, 620, 786

Klessen, R. S., Burkert, A., \& Bate, M. R. 1998, ApJ, 501, L205

Kroupa, P. 1995, MNRAS, 277, 1491

Lada, C. J. 2006, ApJ, 640, L63

Lada, C. J., Bergin, E. A., Alves, J. F., \& Huard, T. L. 2003, ApJ, 586, 286

Lada, C. J., \& Lada, E. A. 2003, ARA\&A, 41, 57

Larson, R. B. 1978, MNRAS, 184, 69 . 1981, MNRAS, 194, 809

Lombardi, M., Alves, J., \& Lada, C. J. 2006, A\&A, 454, 781 (LAL06)

Luhman, K. L. 2004, ApJ, 617, 1216

Matzner, C. D., \& McKee, C. F. 2000, ApJ, 545, 364

McKee, C. F. 1998, in The Origin of Stars and Stellar Systems, ed. C. J. Lada \& N. D. Kylafis (Dordrecht: Kluwer), 29

Motte, F., André, P., Ward-Thompson, D., \& Bontemps, S. 2001, A\&A, 372, L41

Muench, A. A., Lada, C. J. Rathborne, J., Alves, J., \& Lombardi, M. 2008, ApJ, 672, 410

Onishi, T., et al. 1999, PASJ, 51, 871

Rathborne, J. M., Jackson, J. M., \& Simon, R. 2006, ApJ, 641, 389

Rathborne, J. M., Lada, C. J., Muench, A. A., Alves, J., \& Lombardi, M. 2008, ApJS, in press (arXiv: 0708.3660R)

Redman, M., P., Keto, E., Rawlings, J. M. C., \& Williams, D. A. 2004, MNRAS, 352, 1365

Salpeter, E. E. 1955, ApJ, 121, 161

Stanke, T., Smith, M. D., Gredel, R., \& Khanzadyan, T. 2006, A\&A, 447, 609

Shu, F. H. 1977, ApJ, 214, 488

Shu, F. H., Adams, F. C., \& Lizano, S. 1987, ARA\&A, 25, 23

Shu, F. H., Li, Z.-Y., \& Allen, A. 2004, ApJ, 601, 930

Testi, L., \& Sargent, A. I., 1998, ApJ, 508, L91

Walsh, A. J., Myers, P. C., Di Francesco, J., Mohanty, S., Bourke, T. L., Gutermuth, R., \& Wilner, D. 2007, ApJ, 655, 958

Williams, J. P., de Geus, E. J., \& Blitz, L. 1994, ApJ, 428, 693 